\title{
Mobile expandable polystyrene road structure production complex for agribusiness
}

\author{
Nikolay Yermoshin ${ }^{1}$, Dmitriy Filippov ${ }^{2}$, Dmitriy Bukatov ${ }^{2}$, Oleg Biryukov ${ }^{1}$, Dmitriy \\ Simonov ${ }^{1}$, Alexey $\mathrm{Tsyba}^{2}$, and Diana. Kirillova ${ }^{1, *}$ \\ ${ }^{1}$ Peter the Great St.-Petersburg Polytechnic University, 29, Polytechnicheskaya str., 195251, Saint- \\ Petersburg, Russia \\ ${ }^{2}$ Military Academy of Logistics, 8, naberezhnaya Makarova, 199034, Saint-Petersburg, Russia
}

\begin{abstract}
The article addresses the plan of development of a mobile expandable polystyrene road structure production complex. It is necessary to build roads on soils with low bearing capacity in order to ensure the transport accessibility of agribusiness facilities. The proposed solution could be used for the organization of rapid repairs of roads and highways, for eliminating the negative effects caused by natural disasters and other emergencies, for building roads in the arctic zone and ensuring stable functioning of roads in war time. An optimal composition of the process equipment for the mobile expandable polystyrene road structure production complex considered a Plan of construction of a light embankment using EPS-blocks and schematic of an embankment circuit device in various conditions using XPS-plates. The dependence of the time of delivery of the EPS-block objects from the remoteness of construction and feasibility study shows the effectiveness of the use of mobile complexes.
\end{abstract}

\section{Introduction}

The development of the industrial complex and the growth in consumption of raw mineral materials increases the risk of both naturally occurring and technogenic emergencies. Human influence on natural processes is the reason for floods, hurricanes and fires that cause significant damage and cause destruction of infrastructure of communities as well as the transport links connecting them. Most destructive effect is caused by naturally occurring disasters that involve water (floods, mudflows), which decrease the bearing capacity of soil for prolonged periods of time.

Often, due to destruction of bridges or roads, settlements, villages and whole cities become isolated. Such situations require technical and technological solutions for rapid repair of transport links, especially roads and highways [1,2].

\section{Materials and methods}

One of the most perspective ways of rapid repair of roads located on low bearing capacity soil is the building of light embankments. [1,2,3,4].

\footnotetext{
*Corresponding author: kirdiana@mail.ru
} 
Other well-known technological and structural solutions of roadbed construction on weak foundation are expensive in materials, machinery and, most importantly, time, which is unaffordable during emergencies and war time. [3,4,5,6,7].

In applying the light to stabilize the embankments base originate from the condition [6]:

$$
P_{o} \leq P_{b}^{p}
$$

where $P_{o}$ - estimated load; $P_{b}^{p}$ - safe base load.

$$
P_{b}^{p}=\gamma_{n}^{s r} h_{n}
$$

where $\gamma_{n}^{s r}$ - weighted mean of specific embankment weight, satisfying the condition (1); $h_{n}$ - estimated embankment height.

The main principle of constructing and estimating light embankments lies in decreasing the specific load on the weak base by decreasing the weight of the road structure. The decrease in load can be achieved by using materials with significantly lower density than the soil base.

Fig. 1 features a plan demonstrating the principles of constructing a light embankment using expanded polystyrene (EPS) blocks [8, 9, 10, 11,12].

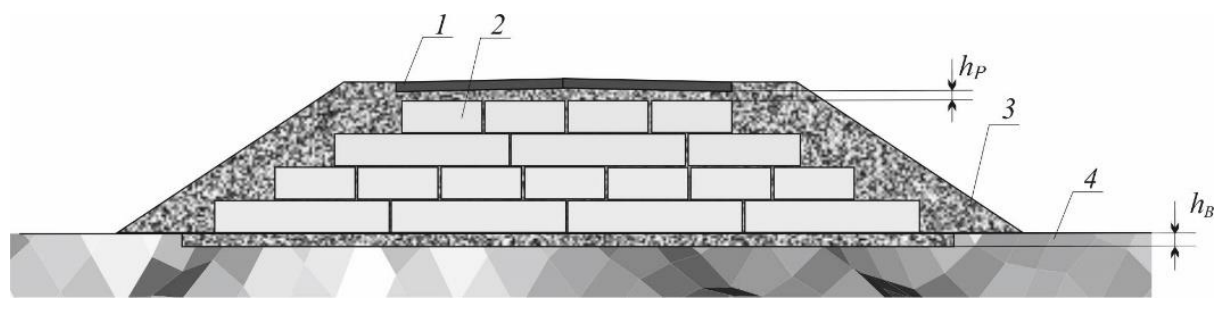

1- road surface; 2- EPS blocks; 3 - sand soil; 4- low bearing capacity soil; $h_{P}$ - load-bearing sand soil layer; $h_{B}$ - equalizing (mounting) sand soil layer.

Fig. 1. Plan of construction of a light embankment using EPS blocks.

Other than EPS blocks, extruded polystyrene (XPS) plates are used in road structures as a cold-resistant layer for regulating water and thermal processes as well as for preventing the subgrade from collapsing. Their use examples are presented on fig. 2, 3, 4 .

The use of such structures is most relevant in the arctic and sub-arctic climate zones, where a modern road is unable to function without proper thermal insulation and man-made structures.

The use of extruded expandable polystyrene for construction, reconstruction and repairs of roads and highways requires efficient technological solutions for the production of EPS blocks and XPS plates.

A mobile expandable polystyrene road structure production complex is therefore proposed $[9,13]$. The proposed complex is a technological extrusive extendable polystyrene (EPS block) production line. 


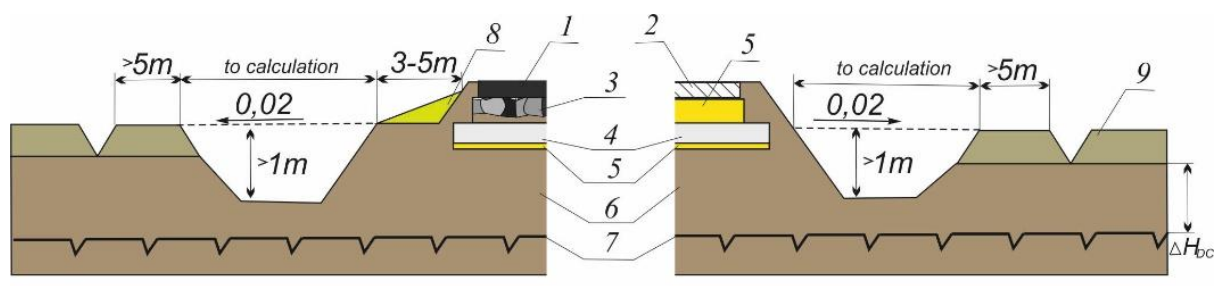

1- asphalt concrete; 2-ferroconcrete (iron-reinforced concrete); 3-tightly bound crushed stones; 4- XPS plate; 5-sand; 6-natural soil; 7- upper bound of permafrost soils; 8-peat powder at the slope; 9-moss and peat; $\Delta H_{D C}$ - active layer thickness.

Fig. 2. A schematic of an embankment made of local clay soil using XPS plates.

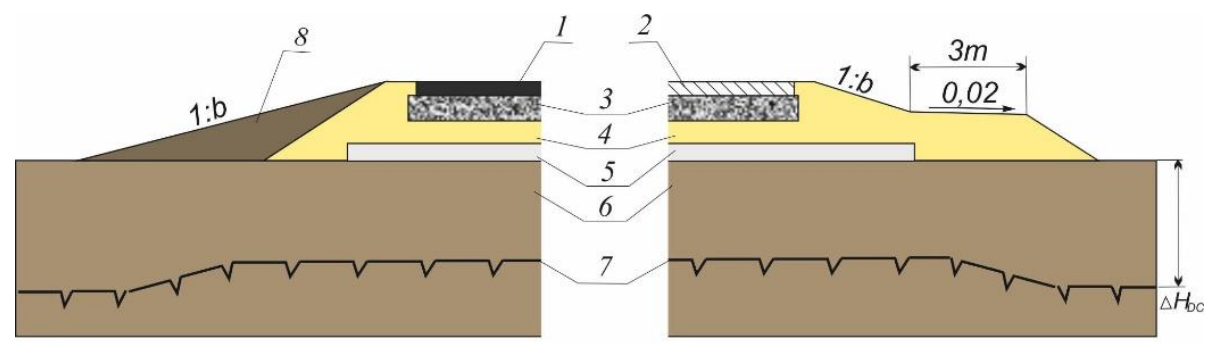

1-asphalt concrete; 2-cement concrete; 3-sand-gravel mixture; 4- sand; 5- XPS plates; 6-natural soil; 7-upper horizon of permafrost soils; 8-imported soil; $\Delta H_{D C}$ - active layer thickness.

Fig. 3. A schematic of an embankment constructed using XPS plates on the soil of subsidence categories IV - V (Russian classification), as well as thawed or loose-frozen and dry-frozen sand soils in the base.

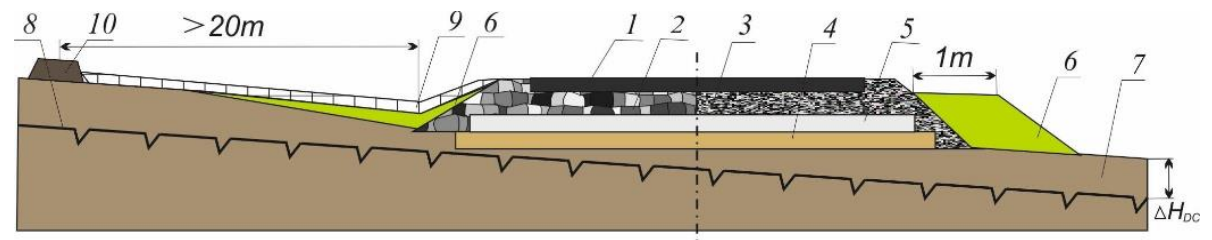

1-asphalt concrete; 2- tightly bound crushed stones; 3-sand-gravel mixture; 4- sand; 5- XPS plates; 6moss and peat; 7-natural soil; 8-upper bound of permafrost soils; 9-pavement; 10-upland roller;

$\Delta H_{D C^{-}}$active layer thickness.

Fig. 4. Schematic of a subgrade in icy sloped slanting areas using XPS plates.

The mobile complex is a set of equipment that produces extendable polystyrene and blocks of it, mounted on car and trailer chassis that allows the supply of materials directly at the venue of roadworks. The machinery and equipment of the complex are shown on fig. 5.

The equipment is mounted onto trailers and cars with high off-road capabilities. The complex is formed according to the principle of modular assembly and can include a varying number of machines and special equipment depending on the given production volumes and conditions of the roadworks.

The complex can be used for production of EPS blocks in absence of a stationary electricity network for when it is necessary to place it as close to the roadworks venue as possible. Particularly, for subgrade restoration, construction of temporary passages and roads on weak soil, construction of roads on swamps and in arctic and sub-arctic zones. A perspective direction for EPS blocks would be their use for repairs of roads during 
emergencies and war time, when the deadlines for repair work are very restricted and the static electricity network either supplies no power or supplies it intermittently $[9-12,14]$.

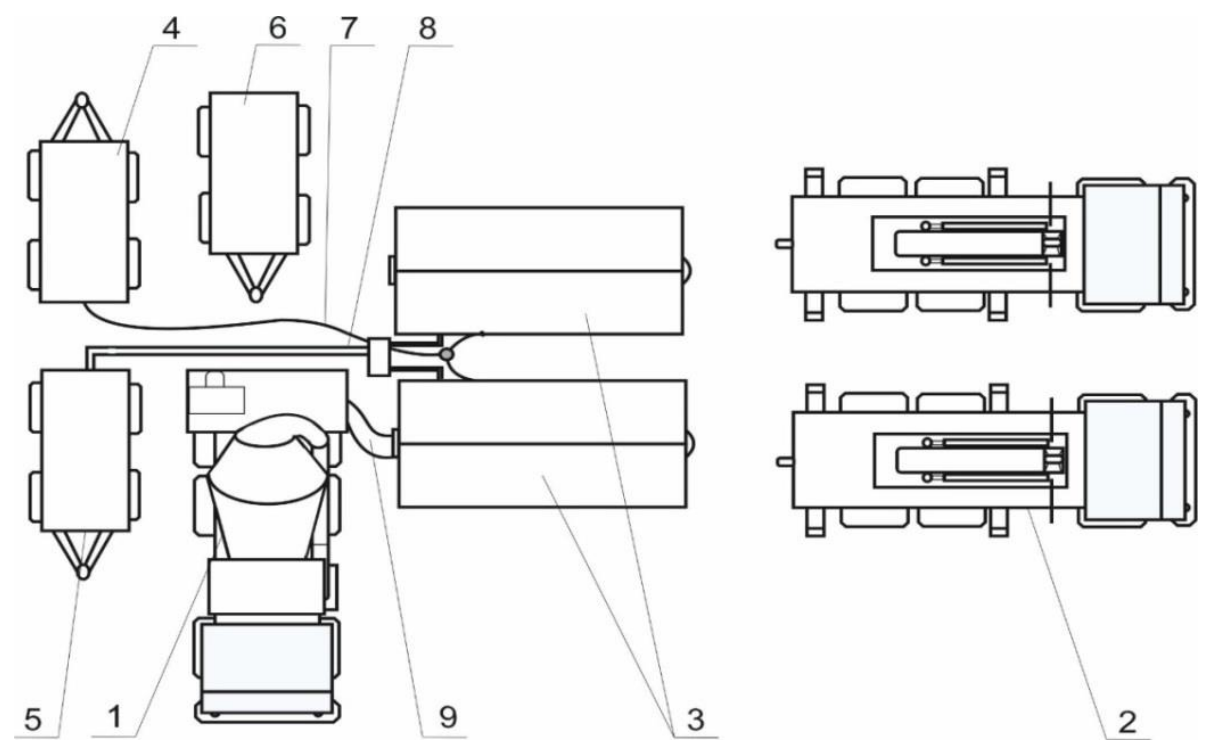

1- car equipped for dry polystyrene foaming; 2- car for block-form transportation (3) car producing extendable polystyrene blocks, equipped with a multilift system, also used for delivering completed blocks to the roadwork venue; 4- diesel power station; 5- compressor station, mounted on a trailer chassis; 6- cargo trailer for material pipe transportation (9), pneumatic pipeline transportation (8) with adjustable flaps, for cable network elements transportation (7) and for transportation of other technological equipment.

Fig. 5. The composition of an extendable polystyrene block-producing complex.

\section{Results}

The primary advantages of the proposed expandable polystyrene road structure production complex are its mobility and autonomy, due to the use of an offroad-capable chassis and the inclusion of a power station, which eliminates the need of having to connect to a stationary electricity network.

Known expandable polystyrene production lines are stationary and require substantial amounts of time to be mounted and configured in case of the production line needs to move.

The technology of producing extendable polystyrene blocks works in such a way that every block exceeds the raw material used for its production by a factor of 30 to 50 in terms of volume. Based on this fact, it is clear that the costs of material delivery to the production venue would be a lot lower compared to delivering a ready product. This can be proven by simple calculations:

$$
T_{V}=T_{\text {Pod }}+t_{\text {Dost }}+t_{U k}+T_{\text {Zav }},
$$

where $T_{V}$ - all time spent on repairing a part of the road; $T_{P o d}$ - preparation work time; $t_{\text {Dost }}$ - EPS block delivery time; $t_{U k}$ - EPS block setting time; $T_{Z a v}$ - concluding work time.

With the rest of the conditions and circumstances being equal, the main indicator which will be measured for a stationary solution and a mobile complex is $t_{\text {Dost }}$ - time needed for 
delivery of a needed number of EPS blocks which depends on the distance between the loading and unloading points, as shown on fig. 6.

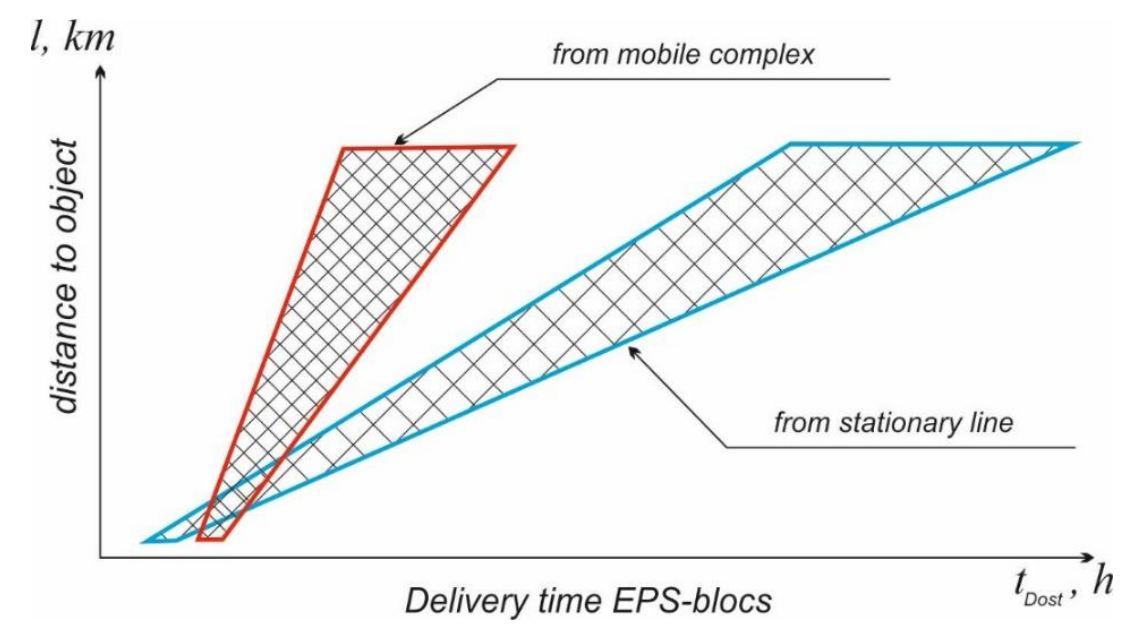

Fig. 6. The graph of delivery time's dependence on the distance to the object unloading point from the distance of production.

\section{Discussion}

The graph above clearly shows that use of a mobile complex significantly cuts the time needed to repair (construct) an area of a road on a weak base.

Another important observation justifying the need for development and use of a mobile expandable polystyrene road structure production complex is its higher economic efficiency as compared to stationary production lines.

Conducting comparative analysis on the repair (construction) costs of an area of a road using a stationary production line and a mobile complex makes it possible to determine the conditions when the use of the latter is more efficient.

With this goal in mind, the total cost of production and delivery of ready-made EPS blocks is considered.

$$
C=\sum_{j}^{m} C_{j P}+\sum_{i}^{n} C_{i D}
$$

where $C$ - total specific roadworks cost; $\sum_{j}^{m} C_{j P}$ - total specific production cost of extendable polystyrene blocks; $\sum_{i}^{n} C_{i D}$ - total specific delivery cost of ready-made blocks to the construction venue.

The dependence of these costs on production volumes is shown on fig. 7 . 


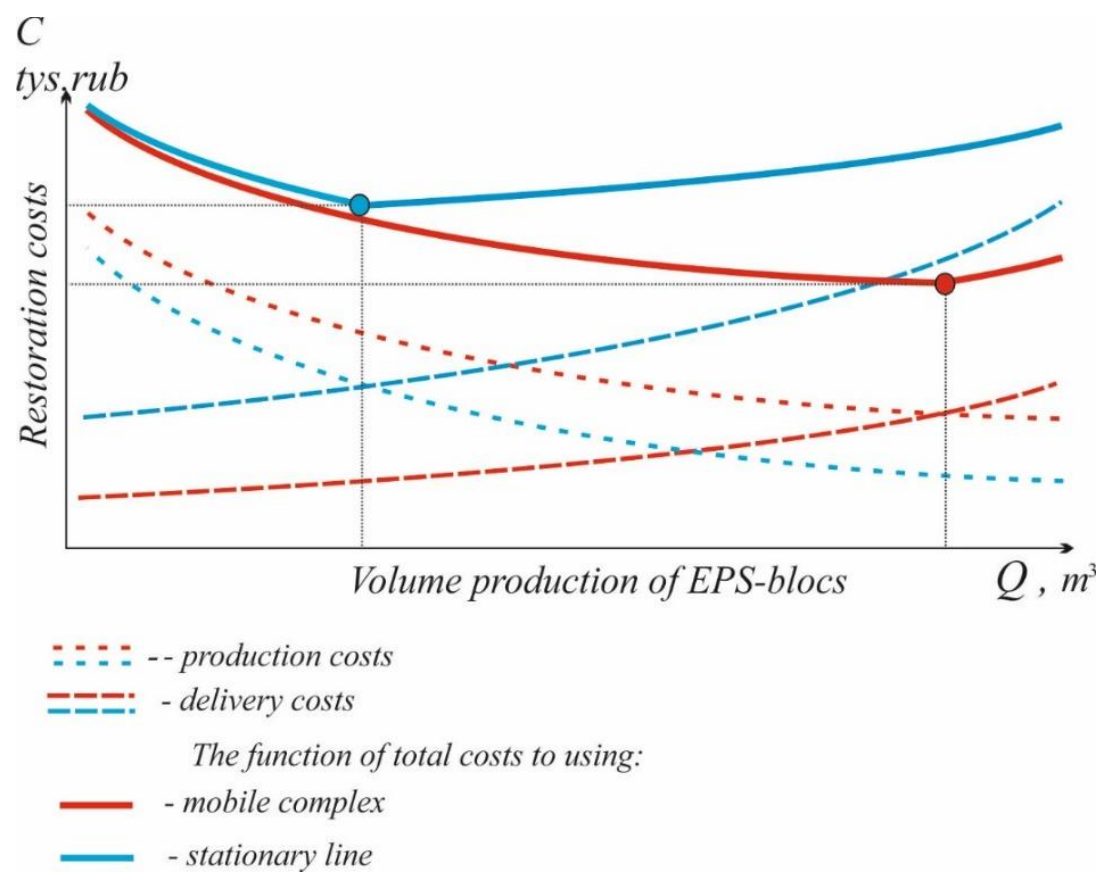

Fig. 7. The cost-volume graph.

As shown on fig. 7 the function of total cost has an optimum which shows the optimal production volume of EPS blocks with minimal total costs.

The comparison of these costs for a stationary line and a mobile complex, by estimation, allows for a conclusion that using mobile solutions for construction and repairs of roads is more advantageous from an economic viewpoint. Additionally, it is necessary to consider shorter extendable polystyrene structures' delivery times.

For developing and integrating the mobile expandable polystyrene road structure production complex into construction, reconstruction and repairs of roads and highways, more theoretical and experimental research is needed.

\section{Conclusion}

The primary goals of this research are determining the technical traits and parameters of technological equipment for production of extrusive extendable polystyrene, evaluating the reliability of functioning of mobile complexes in conditions of emergency, optimizing the technology of production and transportation of products to construction venues etc.

\section{References}

1. B.N. Karpov, M.P. Klekovkina, P.A. Petukhov, Transport 6(49), 19’21 (2013)

2. D.A. Filippov, A process monolithic devices lung embankments. Invention patent №2705282 RF IPC E02D Bull. № 32 (2019)

3. Y.G. Lazarev, E.B. Sinitsyna, S.V. Ugolkov, Technology of transport processes (St. Petersburg state University, Saint-Petersburg, 2016) 
4. B.N. Karpov, A.V. Kvitko, P.A. Petukhov, Collection of materials of the 72th Scientific Conference of professors, teachers, scientists, engineers and graduate students, 7-12 (2016)

5. D. Sheng, Geotechnicque 64(2), 144-154 (2013)

6. S.A. Evtyukov, E.P. Medres, G.A. Ryabinin, A.G. Spector, Construction, calculation and design of lightweight mounds (Petropolis, Saint-Petersburg, 2009)

7. M. Gravit, I. Dmitriev, Y. Lazarev, International Scientific Conference Energy Management of Municipal Facilities and Sustainable Energy Technologies EMMFT 2018 983, 929-938 (2019)

8. M. Gravit, S. Zimin, Y. Lazarev, I. Dmitriev, E. Golub, VIII International Scientific Siberian Transport Forum. Trans Siberia 20191116 (2020)

9. Y. Lazarev, C. Medres, J. Raty, A. Bondarenko, Transportation Research Procedia 20, 396-400 (2017)

10. Y. Lazarev, A. Akhatuly, R. Alzhanova, A. Panfilova, International Scientific Conference Week of Science in SPbPU - Civil Engineering (SPbWOSCE-2015) 53, 01065 (2016)

11. Y. Lazarev, G. Sobko, M. Chakir, Applied Mechanics and Materials 584-586, 16811686 (2014)

12. D.A. Filippov, Method device of light solid embankments. Invention patent №2705282 RF IPC E02D Bull. 32 (2019)

13. D.A. Filippov, N.A. Ermoshin, Y.G. Lazarev, A.T. Zmeyev, Mobile complex for the production of polystyrene foam blocks. Invention patent №2687920 RF IPC B29C 44/02 (2006.01); B60P Bull. 14 (2019)

14. N.A, Ermoshin, A.M. Egoshin, Y.G. Lazarev, A.T. Zmeyev, Issues and methodological aspects of the organization of road activity in the interests of military security of the state (R-COPY, Saint-Petersburg, 2017) 